\title{
Correction to: Accurate and Robust Vanishing Point Detection Method in Unstructured Road Scenes
}

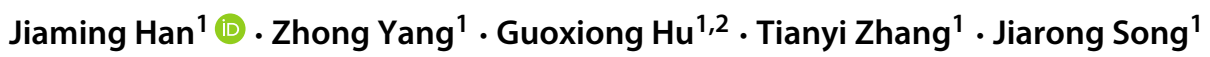

Published online: 14 June 2018

(C) Springer Science+Business Media B.V., part of Springer Nature 2018

\section{Correction to: Journal of Intelligent \& Robotic Systems https://doi.org/10.1007/s10846-018-0814-8}

The original version of this article, unfortunately, contained errors. These are as follows:

1) Formula (6) in the original version should be:

$W_{i j}=\left\{\begin{array}{cc}\exp \left(-p c_{i j}-q h_{i j}\right) & j \in N_{i} \\ 0 & \text { otherwise }\end{array}\right.$

where $\mathbf{p}=\mathbf{1 1}$ and $\mathbf{q}=\mathbf{0 . 5}$. The above formula was used during the experiment of our work. Therefore the data and conclusions of our paper are not affected.

2) Last row of Fig. 3 in the original version was incorrect. The correct Fig. 3 is shown in the following.

3) The running time of the MATLAB version of the $\mathrm{Wu}$ method in both Tables 2 and 4 missed to include "E-1". The correct data for these tables are given in the following:

4) As some of the source codes of the compared methods are not open source, the authors re-program these methods

The online version of the original article can be found at https://doi.org/10.1007/s10846-018-0814-8.

\footnotetext{
$\triangle$ Jiaming Han

hjm@nuaa.edu.cn

Zhong Yang

YZ.NUAA@163.com

Guoxiong $\mathrm{Hu}$

76912412@qq.com

Tianyi Zhang

TianyiZhang@nuaa.edu.cn

Jiarong Song

963514936@qq.com

1 College of Automation Engineering, Nanjing University

of Aeronautics and Astronautics, Nanjing 210016,

People's Republic of China

2 College of Software, Jiangxi Normal University, Nanchang 330022, People's Republic of China

Nanchang 330022, People's Republic of China
}

by themselves. Unfortunately, the authors gave wrong running times of the Ding method and the proposed method. The Wu method is the fastest method and the gLoG method
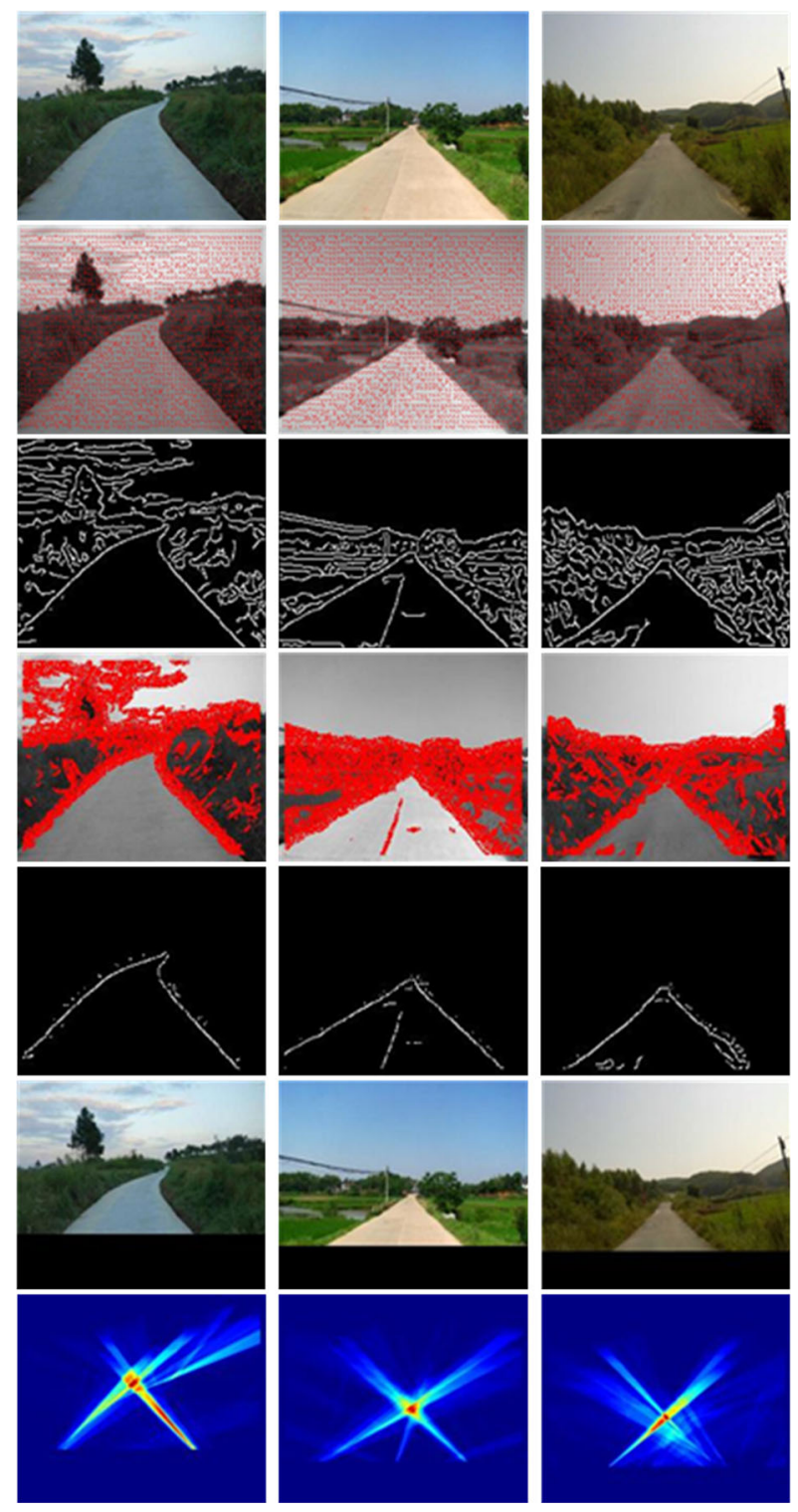

Fig. 3 
Table 2 The average error and average running time

\begin{tabular}{lllllll}
\hline Methods Criterion & Yang [1] & Wu [12] & Moghadam [22] & gLoG [17] & Ding [11] & Ours \\
\hline Average error & 0.0718 & 0.1003 & 0.1421 & 0.0586 & 0.0837 \\
Average running time & $2.7 \mathrm{~s}$ & $1.7 \mathrm{E}-1 \mathrm{~s}$ & $1.2 \mathrm{~s}$ & $47.3 \mathrm{~s}$ & $1.6 \mathrm{~s}$ & 3.0267 \\
\hline
\end{tabular}

Table 4 Running time comparison (per image/s)

\begin{tabular}{lllllll}
\hline Methods Criterion & Yang [1] & Wu [12] & Moghadam [22] & gLoG [17] & Ding [11] & Ours \\
\hline Running time range & $1.7 \sim 4.2$ & $1.1 \mathrm{E}-1 \sim 3.0 \mathrm{E}-1$ & $0.6 \sim 1.5$ & $25.1 \sim 78.3$ & $1.2 \sim 2.8$ & $2.3 \sim 5.8$ \\
Average running time & 3.1 & $1.9 \mathrm{E}-1$ & 1.1 & 44.5 & 1.8 & 3.9
\end{tabular}

is the slowest method. Although the proposed method is not the fastest method and is slower than some of the compared methods, it is quite faster than the gLoG method, which is considered as the state-of-the-art method. Therefore, the proposed method can still be treated as a compromise that has the best performance and a moderate time-consumption. The authors apologize for any inconvenience.

5) In figure 6, the authors use a separate VS program to paint each method's result one by one and unfortunately, column 4, row 2 contains an error in the positioning of the red cross. The correct position of it should be $(135,107)$. The correct image of the whole figure 6 is shown in the following:
Lastly, 6) The bibliographical information for reference 25 is incorrect. The correct reference should read: Yang, C., Zhang, L., Lu, H., Ruan, X. and Yang, M.H., 2013, June. Saliency detection via graph-based manifold ranking. In Computer Vision and Pattern Recognition (CVPR), 2013 IEEE Conference on (pp. 3166-3173). IEEE. Inspired by the reference [24] and [25], we innovatively introduce and improve the manifold ranking method to estimate the roadregion in our work. More details can be seen in the reference $[24,25]$ and our online paper.

Publisher's Note Springer Nature remains neutral with regard to jurisdictional claims in published maps and institutional affiliations.

Fig. 6
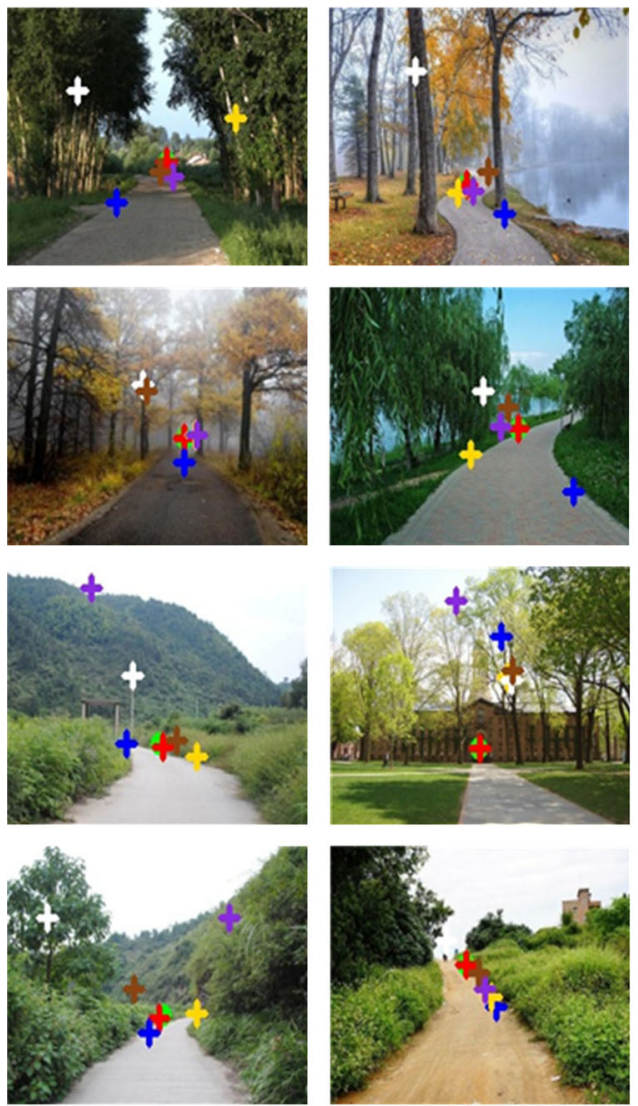
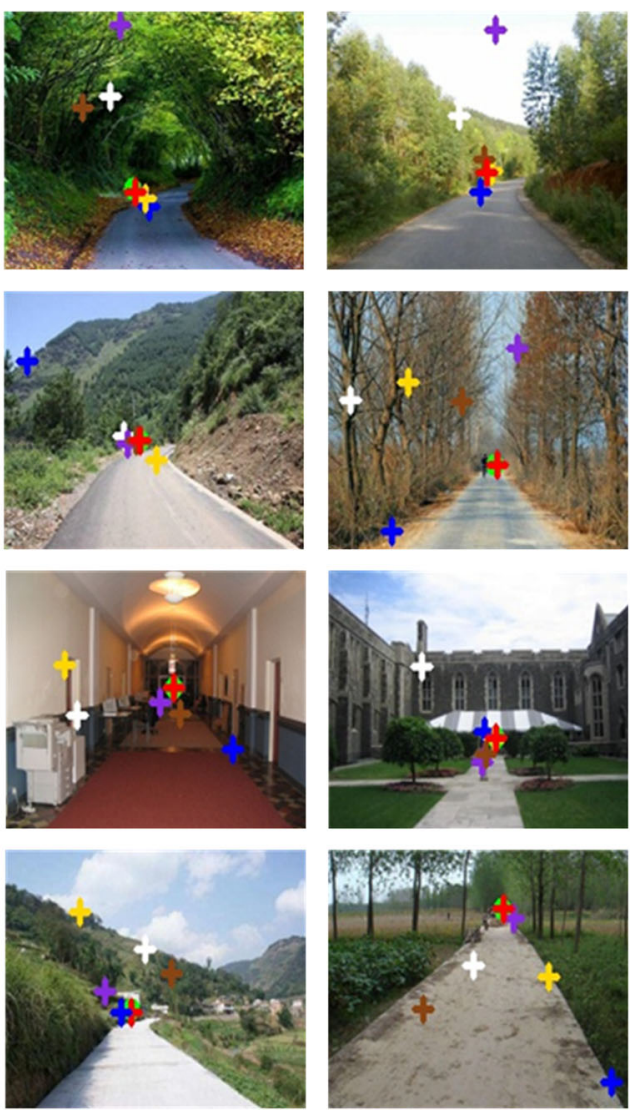